Virginia Commonwealth University vCU Scholars Compass

2011

\title{
Tuning the band gap and magnetic properties of BN sheets impregnated with graphene flakes
}

M. Kan

Peking University

J.Zhou

Peking University

Q. Wang

Peking University, Virginia Commonwealth University

Q.Sun

Peking University, Virginia Commonwealth University

Puru Jena

Virginia Commonwealth University, pjena@vcu.edu

Follow this and additional works at: http://scholarscompass.vcu.edu/phys_pubs

Part of the Physics Commons

Kan, M., Zhou, J., Wang, Q. et al. Tuning the band gap and magnetic properties of BN sheets impregnated with graphene flakes. Physical Review B, 84, 205412 (2011). Copyright (C) 2011 American Physical Society.

\section{Downloaded from}

http://scholarscompass.vcu.edu/phys_pubs/58

This Article is brought to you for free and open access by the Dept. of Physics at VCU Scholars Compass. It has been accepted for inclusion in Physics Publications by an authorized administrator of VCU Scholars Compass. For more information, please contact libcompass@vcu.edu. 


\title{
Tuning the band gap and magnetic properties of $\mathrm{BN}$ sheets impregnated with graphene flakes
}

\author{
M. Kan, ${ }^{1}$ J. Zhou, ${ }^{1}$ Q. Wang, ${ }^{2,3}$ Q. Sun,,${ }^{1,2,3, *}$ and P. Jena ${ }^{3}$ \\ ${ }^{1}$ Department of Materials Science and Engineering, Peking University, Beijing 100871, China \\ ${ }^{2}$ Center for Applied Physics and Technology, Peking University, Beijing 100871, China \\ ${ }^{3}$ Department of Physics, Virginia Commonwealth University, Richmond, VA 23284, USA
}

(Received 22 April 2011; revised manuscript received 19 September 2011; published 11 November 2011)

\begin{abstract}
The BN sheet is a nonmagnetic wide-band-gap semiconductor. Using density functional theory, we show that these properties can be fundamentally altered by embedding graphene flakes. Not only do graphene flakes preserve the two-dimensional (2D) planar structure of the BN sheet, but by controlling their shape and size, unexpected electronic and magnetic properties also emerge. The electronic band structure can be tuned from a direct gap to an indirect gap, the energy gap can be further modulated by changing the bonding patterns, and both hole injecting or electron injecting can be achieved by tailoring the triangular embedding pattern. Furthermore, the Lieb theorem still holds, and the embedded triangular graphene flakes become ferromagnetic with full spin polarizations of the introduced electrons or holes, opening the door to their use as spin filters. The study sheds new light on hybrid single-atomic-layer engineering for unprecedented applications of 2D nanomaterials.
\end{abstract}

DOI: 10.1103/PhysRevB.84.205412

PACS number(s): 31.15.E-, 36.40.Cg

\section{INTRODUCTION}

Synthesis of graphene, a single layer of $\mathrm{sp}^{2}$ hybridized carbon atoms, by mechanical peeling ${ }^{1}$ and epitaxial growth ${ }^{2}$ and their unique proprieties, ${ }^{3-10}$ such as high electron mobility, ambipolar effect, Klein tunneling, two-dimensional (2D) massless Dirac fermions, and anomalous quantum Hall effect, have invoked a great interest in the search of similar materials. Among them, BN sheets have attracted considerable attention. These are synthesized through the chemically derived route ${ }^{11}$ by thermal decomposition of borazine on the surface of a transition metal or by micromechanical cleavage technique. ${ }^{12}$ However, unlike graphene, the $\mathrm{BN}$ sheet $(h-\mathrm{BN})$ is a wideband-gap semiconductor with a direct gap of $4.64 \mathrm{eV} .^{13-15}$ Tuning this energy gap for potential applications has been a challenge. Although several techniques such as hydrogenation, fluorination, functional group adsorption, and metal doping have been used, ${ }^{14,16}$ these lead to the deformation of the pristine 2D planar structure of the BN sheet due to either the $\mathrm{sp}^{3}$ bonding or the distortion caused by size mismatch between metal atom and the $\mathrm{B} / \mathrm{N}$ atom. This has led to an interesting question: Is it possible to tune the electronic structure and magnetic properties of a $\mathrm{BN}$ sheet, while keeping its $2 \mathrm{D}$ planar geometry?

In this paper we address this challenge. One option is to replace some of the $\mathrm{BN}$ atoms by grafting another planar structure with very similar bond length but with different composition. Among all the single-atomic layers studied thus far, graphene flakes are the only candidates, because a graphene sheet and a BN sheet have the same geometry and almost the same bond length. In fact several researchers have used this property to modify the electronic structure of a graphene sheet by embedding BN flakes. For example, using a thermal catalytic chemical vapor decomposition (CVD) method, $\mathrm{Ci}$ et al. have successfully synthesized a BN-flakeembedded graphene sheet, ${ }^{17}$ in which all of the $\mathrm{B}$ and $\mathrm{N}$ atoms form $\mathrm{BN}$ pairs with each other, and the $\mathrm{C}$ atoms occupy other sites of the hexagonal lattice. Such a kind of hybridized sheet has also been studied in a recent theoretical work. $^{18,19}$ Because graphene is semimetallic, and graphene flakes can be magnetic, we hypothesized that it may be possible to modulate the electronic and magnetic properties by introducing graphene flakes into a BN sheet. We address the following questions: How does the energy band gap change with the shape, size, and concentration of graphene flakes? How can the magnetic properties be tuned? According to the Lieb theorem, ${ }^{20}$ free-standing triangular graphene flakes are magnetic because of the different numbers of $\mathrm{C}$ atoms in the $\alpha$ and $\beta$ sublattices. Can the magnetism of graphene flakes be kept when embedded in a BN sheet? Does the Lieb theorem still hold for the embedded cases? How are the B or N atoms in the $\mathrm{BN}$ sheet matrix polarized?

To answer the above questions, we carried out firstprinciples calculations of the electronic structure and magnetic properties of a BN sheet embedded with graphene flakes of different size and shape. Our results show that the midgap states $^{18}$ are introduced when graphene flakes are embedded, thus reducing the energy gap. The number of midgap states depends on the geometric shape and size of the graphene flakes, providing very flexible ways to modulate the electronic structures of a BN sheet. Furthermore, we found triangular graphene flakes can induce spin polarization in the BN sheet, and the total magnetic moment can be tuned by changing the size of the graphene flakes. Very recently, Wei et al. have experimentally demonstrated one efficient method to insert $\mathrm{C}$ into the BN hexagonal sheet and nanoribbons with the help of in situ high-energy electron beam irradiation-induced vacancies, which may overcome the uncontrollable hybridized pattern in Ref. 17 and obtain a well-ordered embedding pattern. ${ }^{21}$

\section{COMPUTATIONAL METHOD}

Calculations are based on spin-polarized density functional theory (DFT) using generalized gradient approximation $(\mathrm{GGA})^{22}$ for exchange-correlation potential prescribed by Perdew-Burke-Ernzerhof (PBE) and the DMOL3 package. ${ }^{23}$ Periodic boundary condition is used to simulate $2 \mathrm{D}$ infinite sheet. For the geometric and electronic structural calculations, a supercell containing of 96 atoms is used, as shown in 


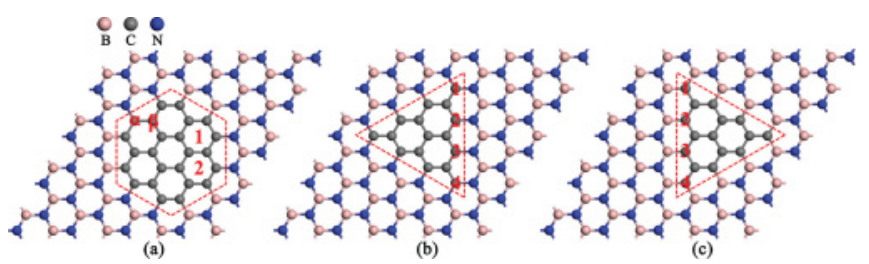

FIG. 1. (Color online) Optimized geometries of unit cells of graphene flake-impregnated $\mathrm{BN}$ sheets. (a) hexagonal graphene flakes $\mathrm{H}-\mathrm{C}_{12} \mathrm{C}_{12}(\alpha=\beta)$, (b) triangular graphene flakes $\mathrm{T} 1-\mathrm{C}_{10} \mathrm{C}_{6}(\alpha>\beta)$ with $\mathrm{B}$ edges, (c) triangular graphene flakes $\mathrm{T} 2-\mathrm{C}_{6} \mathrm{C}_{10}(\alpha<\beta)$ with $\mathrm{N}$ edges.

Figs. 1(a)-1(c). We used a vacuum space of $15 \AA$ in the direction normal to the sheet in order to avoid interactions between two images. The Brillouin zone is represented through Monkhorst-Pack special $k$-point scheme ${ }^{24}$ with $7 \times$ $7 \times 1$ grid mesh. We used effective core potential with double-numerical plus polarization (DNP) basis set, which includes a polarization $p$-function on all hydrogen atoms. All the structures are relaxed without any symmetric constraints. The criteria of convergence of energy, force, and displacement are set as $1 \times 10^{-5} \mathrm{Ha}, 0.002 \mathrm{Ha} / \AA$, and $0.005 \AA$, respectively. The accuracy of our procedure was tested using pristine $h$-BN sheet. The optimized bond length of B-N is $1.446 \AA$, which agrees well with experimental value of $1.45 \AA$. The calculated energy gap of $\mathrm{BN}$ sheet, $4.71 \mathrm{eV}$, also fits well with previous theoretical result. ${ }^{14,25}$

The honeycomb lattice sheet is made up of two sublattices, which are marked as $\alpha$ and $\beta$ sublattices in Fig. 1. In our study we designate the $\mathrm{B}$ atoms in the $\mathrm{BN}$ sheet as belonging to the $\alpha$ sublattice, while the $\mathrm{N}$ atoms belong to the $\beta$ sublattice. Hence, those $\mathrm{C}$ atoms replacing the $\mathrm{B}$ or $\mathrm{N}$ will be in the $\alpha$ sublattice or $\beta$ sublattice, respectively. Three types of hybridized structures with different shapes are considered: (1) hexagonal graphene flakes [Fig. 1(a), which we refer to as $\mathrm{H}-\mathrm{C}_{\alpha} \mathrm{C}_{\beta}, \alpha=\beta$, where the $\alpha$ or $\beta$ defines the number of the $\mathrm{C}$ atoms in the $\alpha$ or $\beta$ sublattice], (2) triangular graphene flakes with $\alpha>$ $\beta$ [Fig. 1(b), T1- $\mathrm{C}_{\alpha} \mathrm{C}_{\beta}$ ], and (3) triangular graphene flakes with $\alpha<\beta$ [Fig. 1(c), T2- $\mathrm{C}_{\alpha} \mathrm{C}_{\beta}$ ]. Note that in the T1- $\mathrm{C}_{\alpha} \mathrm{C}_{\beta}$ configuration, the graphene flake is connected to the $\mathrm{BN}$ sheet through $\mathrm{C}-\mathrm{N}$ bonds at edges and vertices, while for $\mathrm{T} 2-\mathrm{C}_{\alpha} \mathrm{C}_{\beta}$, the connections are through $\mathrm{C}-\mathrm{B}$ bonds.

\section{RESULTS AND DISCUSSIONS}

\section{A. Geometric Structures}

For $\mathrm{H}-\mathrm{C}_{\alpha} \mathrm{C}_{\beta}$, the total number of $\mathrm{C}$ atoms is $(\alpha+\beta)$, which equals to $6^{*} m^{2}$, where $m$ is the number of the hexatomic rings on each edge of the hexagonal graphene flake. For example, each edge corresponds to two hexatomic rings in Fig. 1(a), so the total number of $\mathrm{C}$ atoms in this structure is $6 \times 2^{2}=$ 24. For triangular configurations, $\mathrm{T} 1-\mathrm{C}_{\alpha} \mathrm{C}_{\beta}$ and $\mathrm{T} 2-\mathrm{C}_{\alpha} \mathrm{C}_{\beta}$, the total number of $\mathrm{C}$ atoms is also $(\alpha+\beta)$, which equals to $n^{2}$, where $n$ is the number of the $\mathrm{C}$ atoms on each edge of the triangular graphene flake. For example, each edge consists of four $\mathrm{C}$ atoms in Fig. 1(b), so the total number of $\mathrm{C}$ atoms here is $4^{2}=16$. It is interesting to note that $n$ also equals to $|\alpha-\beta|$. We determine the cell lattice constant of hybrid structures according to the concentrations of $\mathrm{C}$ and $\mathrm{BN}$. The unit cell lattice constant in our calculation is determined by the concentration of graphene flakes, and the following formula is used:

$a=\sqrt{\left(12 \times a_{\mathrm{BN}}\right)^{2} \times \frac{96-(\alpha+\beta)}{96}+\left(12 \times a_{\mathrm{gra}}\right)^{2} \times \frac{\alpha+\beta}{96}}$,

where 96 is the total number of atoms in the studied supercell, and $a_{\mathrm{BN}}$ and $a_{\text {gra }}$ are the lattice constants of pure $h$-BN and graphene, respectively. The accuracy of the formula is tested using $\mathrm{H}-\mathrm{C}_{12} \mathrm{C}_{12}$. The optimized unit cell lattice constant of $\mathrm{H}-\mathrm{C}_{12} \mathrm{C}_{12}$ is $17.40 \AA$, which is equal to the calculated one. It should be pointed out that because of the small difference between $a_{\mathrm{BN}}$ and $a_{\text {gra }}$, a small strain would be induced if using $a_{\mathrm{BN}}$ as the lattice constant for the hybrid sheet, which will affect the properties of the system.

When the hexagonal graphene flake is embedded in a BN sheet, structural optimization, as expected, shows that the 2D planar geometry is kept. The C-B bonds lie in the range of 1.514 to $1.539 \AA$, and the $\mathrm{C}-\mathrm{N}$ bonds are between 1.393 and $1.427 \AA$. These values are in good agreement with previous theoretical results. ${ }^{18}$ In addition, for $m=1,2$, and 3, the C-B (C-N) bond lengths are $\sim 1.51, \sim 1.52$, and $\sim 1.53 \AA(\sim 1.43$, $\sim 1.41$, and $\sim 1.40 \AA$ ), respectively, suggesting that the C-B (C-N) bond lengths only slightly change as the size of the hexagon graphene flakes increase. For systems with triangular graphene flakes, the $\mathrm{C}-\mathrm{B}$ bond lengths are $\sim 1.52 \AA$ at edges and $\sim 1.54 \AA$ at vertices for $\mathrm{T} 1-\mathrm{C}_{\alpha} \mathrm{C}_{\beta}$, while the $\mathrm{C}-\mathrm{N}$ bond lengths are $\sim 1.41 \AA$ at edges and $\sim 1.42 \AA$ at vertices for T2- $\mathrm{C}_{\alpha} \mathrm{C}_{\beta}$. And they also change slightly with the size of the triangular graphene flakes. In addition, the $\mathrm{C}-\mathrm{C}$ bond length and $\mathrm{B}-\mathrm{N}$ bond length in our optimized structures range from 1.392 to $1.438 \AA$ and 1.432 to $1.469 \AA$, which are close to the values of pristine graphene and $\mathrm{BN}$ sheet, namely, 1.420 and $1.450 \AA$ A, respectively.

\section{B. Electronic Structures}

The calculated band structures of $\mathrm{H}-\mathrm{C}_{\alpha} \mathrm{C}_{\beta}$ are plotted in Fig. 2(a) for $m=1,2$, and 3, respectively. We see that all $\mathrm{H}-\mathrm{C}_{\alpha} \mathrm{C}_{\beta}$ systems are direct band-gap semiconductors with the highest occupied crystalline orbitals (HOCOs) and lowest unoccupied crystalline orbitals (LUCOs) located at the $\Gamma$ point of the reciprocal space. The HOCO and LUCO are both mostly contributed by the $\mathrm{C}$ atoms in the hexagonal graphene flakes as show in Fig. 2(a). The energy gaps of the $\mathrm{H}-\mathrm{C}_{\alpha} \mathrm{C}_{\beta}$ structures for $m=1,2$, and 3 are 3.56, 2.29, and $1.44 \mathrm{eV}$, respectively, suggesting that the energy gap decreases when the size $(m)$, or the impregnating concentration, of the hexagonal graphene flake increases [Fig. 2(b)], and can be continuously tuned from a BN-like band gap $(4.71 \mathrm{eV})$ to a graphene-like band gap $(0 \mathrm{eV})$. Furthermore, we find that the band gap dependence of $m$ is linear, namely, $E_{g}=4.71-m^{*} 4.71 / 4=4.71-1.18^{*} m$, as shown in Fig. 2(b). For $(5 \times 5)$ unit cells, we find the band gap changing as $E_{g}=4.71-m^{*} 4.71 / 5$. So we can extend the formula to $E_{g}=4.71-m^{*} 4.71 / \mathrm{N}$ for $(N \times N)$ unit cells. The concentration of carbon can be tuned by changing the size of the super cell, which would affect the electronic structure of the system. For example, when a $(4 \times 4)$ supercell is increased 


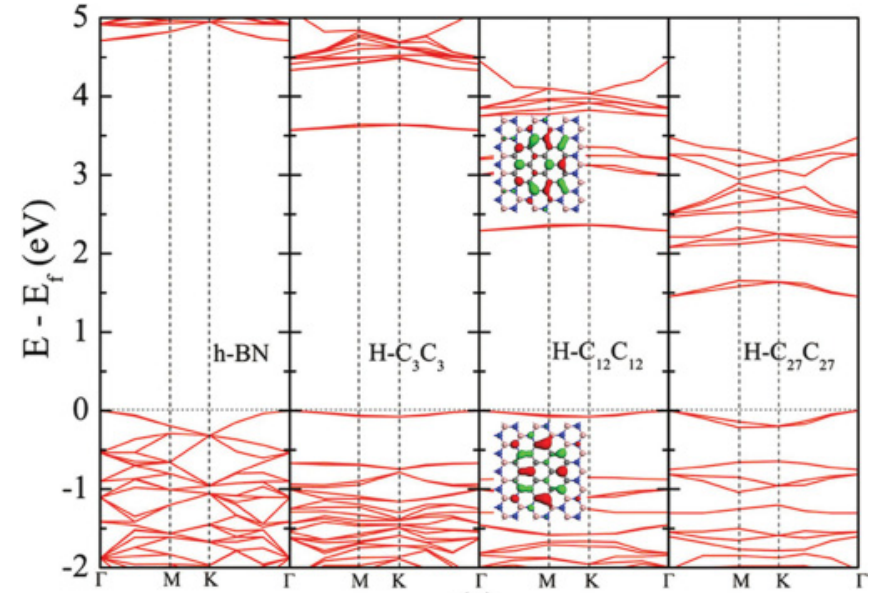

(a)

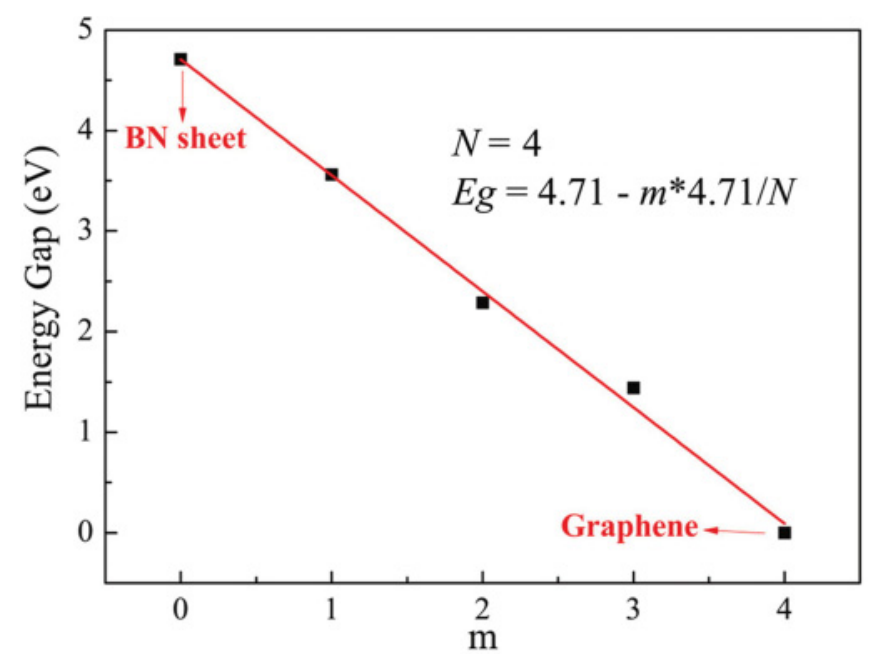

(b)

FIG. 2. (Color online) (a) Calculated band structures for h-BN, H$\mathrm{C}_{3} \mathrm{C}_{3}, \mathrm{H}-\mathrm{C}_{12} \mathrm{C}_{12}$, and $\mathrm{H}-\mathrm{C}_{27} \mathrm{C}_{27}$, respectively. (b) Changes of energy band gap $E_{g}$ of $\mathrm{H}-\mathrm{C}_{\alpha} \mathrm{C}_{\alpha}$ with respect to $m\left[=(\alpha / 3)^{1 / 2}\right]$.

to $(5 \times 5)$, the energy bands near the Fermi level become much more dispersed.

Because C-C bonding is stronger than C-B and C-N bonding, while the hexagonal configuration has more $\mathrm{C}-\mathrm{C}$ bonds as compared to the triangular ones, accordingly the former is energetically more stable. However, the growth pattern of materials can be changed by kinetics. A recent study ${ }^{26}$ suggests that both hexagonal and triangular configurations of graphene flakes embedded in a BN sheet can exist under different chemical potentials. Thus, we also study the energy band structures of the triangular graphene flakes with $n=2,3$, 4, and 5 embedded, respectively in BN sheet [Fig. 3(a)]. The energy band structures indicate that $\mathrm{T} 1-\mathrm{C}_{\alpha} \mathrm{C}_{\beta}$ structures are indirect band-gap semiconductors. And the number of midgap states is $2,3,4$, and 5 for $\mathrm{T} 1-\mathrm{C}_{3} \mathrm{C}_{1}, \mathrm{~T} 1-\mathrm{C}_{6} \mathrm{C}_{3}, \mathrm{~T} 1-\mathrm{C}_{10} \mathrm{C}_{6}$, and T1- $\mathrm{C}_{15} \mathrm{C}_{10}$, respectively, which equals $n(=|\alpha-\beta|)$ in each case. This is due to the different numbers of valence electrons of $\mathrm{B}$, $\mathrm{C}$, and $\mathrm{N}$ atoms.

The substitution of one $\mathrm{B}$ atom by one $\mathrm{C}$ atom will bring one more electron into the structure, while the substitution of one $\mathrm{N}$ by one $\mathrm{C}$ will introduce one hole. For $\mathrm{T} 1-\mathrm{C}_{\alpha} \mathrm{C}_{\beta}$, the

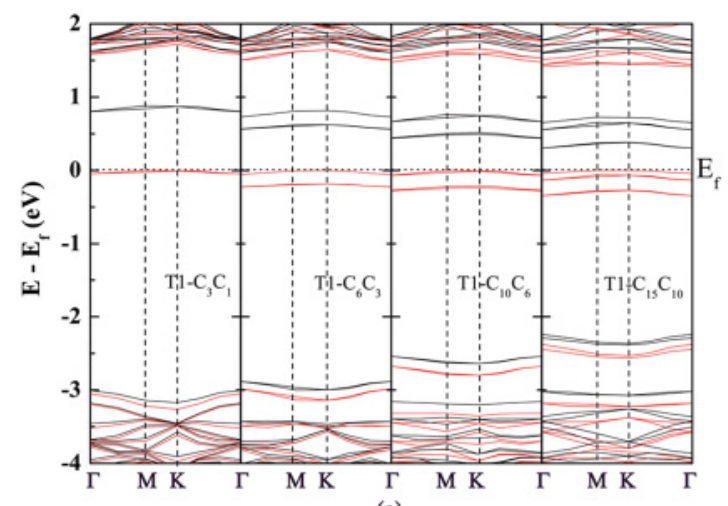

(a)

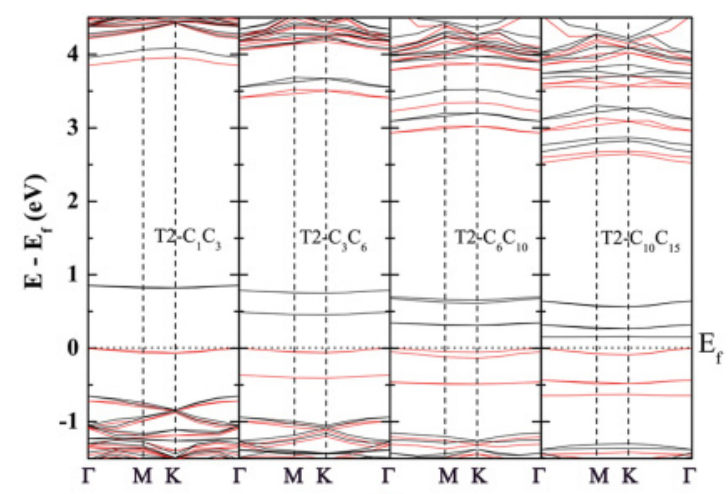

(b)

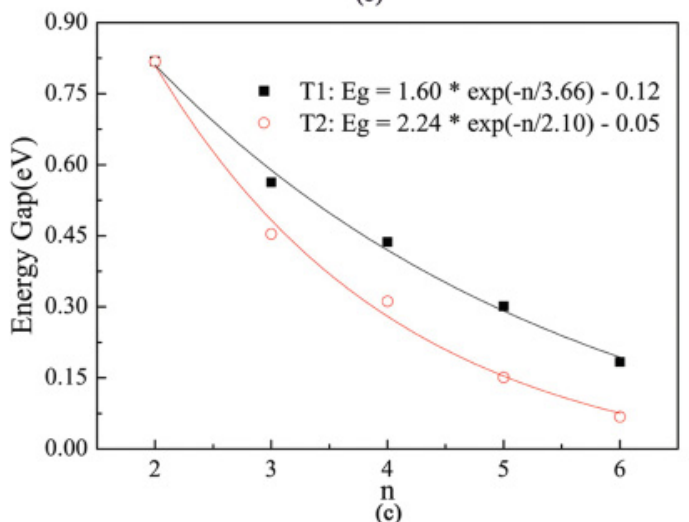

FIG. 3. (Color online) Calculated band structures of $\mathrm{T} 1-\mathrm{C}_{\alpha} \mathrm{C}_{\beta}$ and T2- $\mathrm{C}_{\alpha} \mathrm{C}_{\beta}$; the red and black lines represent the spin-up and spin-down states, respectively. (a) Calculated band structures for $\mathrm{T} 1-\mathrm{C}_{1} \mathrm{C}_{3}$, T1- $\mathrm{C}_{3} \mathrm{C}_{6}, \mathrm{~T} 1-\mathrm{C}_{6} \mathrm{C}_{10}$, and $\mathrm{T} 1-\mathrm{C}_{10} \mathrm{C}_{15}$, respectively. (b) Calculated band structures for $\mathrm{T} 2-\mathrm{C}_{3} \mathrm{C}_{1}, \mathrm{~T} 2-\mathrm{C}_{6} \mathrm{C}_{3}, \mathrm{~T} 2-\mathrm{C}_{10} \mathrm{C}_{6}$, and $\mathrm{T} 2-\mathrm{C}_{15} \mathrm{C}_{10}$, respectively. (c) Energy band gap $E_{g}$ of T1- $\mathrm{C}_{\alpha} \mathrm{C}_{\beta}$ and $\mathrm{T} 2-\mathrm{C}_{\alpha} \mathrm{C}_{\beta}$ with respect to $n$.

number of replaced $\mathrm{B}$ atoms is $\left(n^{2}+n\right) / 2$, and the number of replaced $\mathrm{N}$ atoms is $\left(n^{2}-n\right) / 2$. It means that a graphene flake will bring $\left(n^{2}+n\right) / 2$ electrons and $\left(n^{2}-n\right) / 2$ holes into a T1- $\mathrm{C}_{\alpha} \mathrm{C}_{\beta}$ structure, and the net effect is hole injecting with $n$ electrons being introduced. These will occupy the unoccupied bands contributed mainly by the $2 p$-electron states of B atoms. Thus, the resulting doping states appear close to the conduction bands as shown in Fig. 3, where the red lines in the energy band structures correspond to the spin-up states and the black lines to the spin-down states. In the impregnated material of T1- $\mathrm{C}_{\alpha} \mathrm{C}_{\beta}$, all spin-up states are occupied, while all spin-down states lie 
above the Fermi level, resulting in a magnetic moment of $n \mu_{B}$. Hence, the $\mathrm{T} 1-\mathrm{C}_{\alpha} \mathrm{C}_{\beta}$ systems are magnetically different from the pristine $h$ - $\mathrm{BN}$ sheet and hybrid $\mathrm{H}-\mathrm{C}_{\alpha} \mathrm{C}_{\beta}$ sheet, which are nonmagnetic. The calculated energy gap of $\mathrm{T} 1-\mathrm{C}_{\alpha} \mathrm{C}_{\beta}$ changes with the size (n) of graphene flakes. This is shown in Fig. 3(c), which indicates that the energy gap decreases as the sizes of the graphene flake increases, similar to what happens in $\mathrm{H}-\mathrm{C}_{\alpha} \mathrm{C}_{\beta}$.

In the $\mathrm{T} 1-\mathrm{C}_{\alpha} \mathrm{C}_{\beta}$ configuration, the graphene flake connects with the $\mathrm{BN}$ sheet through $\mathrm{C}-\mathrm{N}$ bonding. This connection can also be achieved through $\mathrm{C}-\mathrm{B}$ bonding, which results in a T2- $\mathrm{C}_{\alpha} \mathrm{C}_{\beta}$ configuration. Because C-B bonding is stronger than $\mathrm{C}-\mathrm{N}$ bonding, in $\mathrm{T} 1$ and $\mathrm{T} 2$ configurations, the connections between the BN and graphene flake are through B-C and B-N bonding, respectively, hence the $\mathrm{T} 1$ configuration is more stable than T2. Recently Natalia et al. ${ }^{27}$ reported that the energy required to "swap" a B atom with a $\mathrm{C}$ is lower than that of swapping an $\mathrm{N}$ with a $\mathrm{C}$ atom in a positively charged condition, while the negative charge gives the opposite result. This means that both $\mathrm{T} 1-\mathrm{C}_{\alpha} \mathrm{C}_{\beta}$ and $\mathrm{T} 2-\mathrm{C}_{\alpha} \mathrm{C}_{\beta}$ configurations can exist in different experimental environments.

Following the similar procedure as discussed above for the T1- $\mathrm{C}_{\alpha} \mathrm{C}_{\beta}$ configuration, we studied the electronic structure and magnetic properties for the $\mathrm{T} 2-\mathrm{C}_{\alpha} \mathrm{C}_{\beta}$ configuration. The band structures for different sizes are given in Fig. 3(b), and the changes of the energy gap with size are shown in Fig. 3(c). We see both similarities and differences with the results in the T1- $\mathrm{C}_{\alpha} \mathrm{C}_{\beta}$ configuration. For example, the $\mathrm{T} 2-\mathrm{C}_{\alpha} \mathrm{C}_{\beta}$ structure is also a magnetic semiconductor with an indirect energy gap, which decreases with the size of the graphene flake because of the introduced doping states. However, in $\mathrm{T} 2-\mathrm{C}_{\alpha} \mathrm{C}_{\beta}$, the number of substituted $\mathrm{B}$ sites is $\left(n^{2}-n\right) / 2$, while the number of substituted $\mathrm{N}$ sites is $\left(n^{2}+n\right) / 2$, therefore, $n$ holes are introduced to the system. Embedding graphene flakes in this configuration is equivalent to electron injecting. Therefore, the doping states would be located near the valence bands of pristine $h$-BN sheet. We can also see that electron injecting (T2- $\left.\mathrm{C}_{\alpha} \mathrm{C}_{\beta}\right)$ is more efficient than hole injecting $\left(\mathrm{T} 1-\mathrm{C}_{\alpha} \mathrm{C}_{\beta}\right)$ in modulating the energy band gap.

\section{Magnetic Properties of Triangular embeddings}

From the band structures we see that although both the pristine 2D BN sheet and graphene sheet are nonmagnetic, the embedded system can be spin polarized. In this section, we discuss magnetism in more detail. We find that the spin polarization of the system can be induced and tuned not only by changing the shape and size of graphene flakes but also by changing the embedding bonds either through $\mathrm{C}-\mathrm{N}$ or C-B. For example when hexagonal graphene flakes are embedded in a BN sheet, the system remains nonmagnetic, because isolated hexagonal graphene flakes themselves are nonmagnetic. The situation is different for triangular graphene flakes, where different numbers in the $\alpha$ and $\beta$ sublattices make the triangular flakes themselves magnetic in accordance with the Lieb theorem. ${ }^{20}$ When embedded in a BN sheet, the magnetism can still be kept, and the Lieb theorem holds. This is different from what happens in some transition metal atoms. For instance, a Ni atom in a free state has the magnetic moment of $2 \mu_{B}$, however, when embedded in a BN sheet,

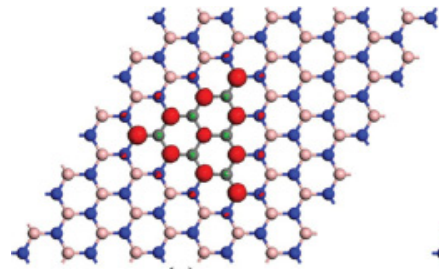

(a)

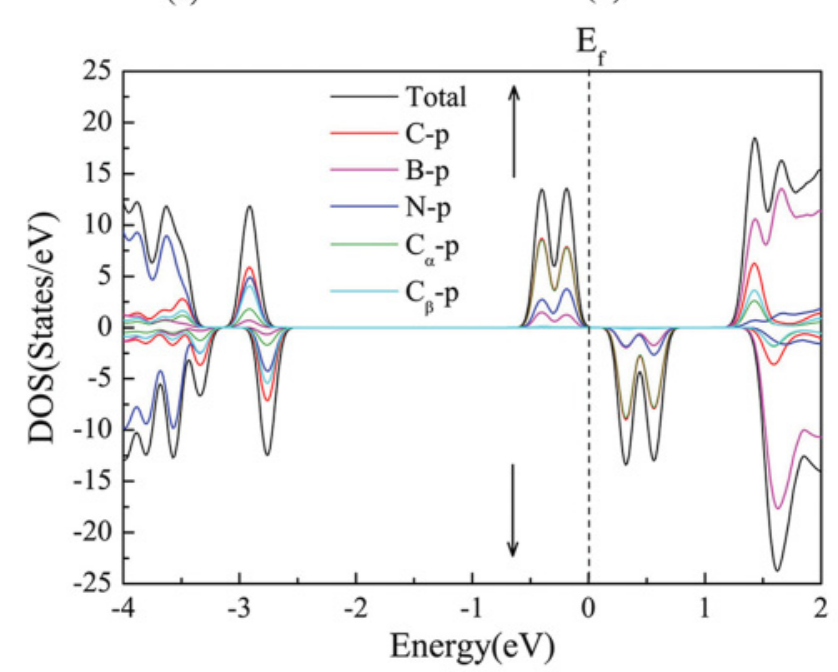

(c)

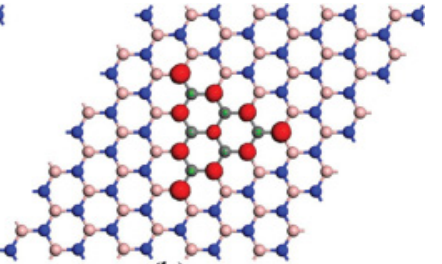

(b)
FIG. 4. (Color online) Spin density isosurface of (a) $\mathrm{T} 1-\mathrm{C}_{10} \mathrm{C}_{6}$ and (b) T2- $\mathrm{C}_{6} \mathrm{C}_{10}$ with the value of 0.055 electron $/ \AA^{3}$. (c) Projected density of states of $\mathrm{T} 1-\mathrm{C}_{10} \mathrm{C}_{6}$.

the magnetic moment is totally quenched. ${ }^{16}$ From this point of view, we can see that the $\mathrm{BN}$ sheet is an ideal substrate to assemble magnetic triangular graphene flakes. To see how the spins are distributed and how the substrate of the $\mathrm{BN}$ sheet is polarized, we plot the spin density of $\mathrm{T} 1-\mathrm{C}_{10} \mathrm{C}_{6}$ and $\mathrm{T} 2-\mathrm{C}_{6} \mathrm{C}_{10}$ as shown in Figs. 4(a) and 4(b). The energy difference $(\Delta \mathrm{E}=$ $\left.\mathrm{E}_{M}-\mathrm{E}_{N M}\right)$ for $\mathrm{T} 1-\mathrm{C}_{10} \mathrm{C}_{6}$ and $\mathrm{T} 2-\mathrm{C}_{6} \mathrm{C}_{10}$ are found to be -453 $\mathrm{meV}$ and $-239 \mathrm{meV}$, respectively, where $E_{M}$ is the energy of magnetic state and $E_{N M}$ the energy of nonmagnetic state. We can see that a large difference exists between configurations $\mathrm{T} 1$ and $\mathrm{T} 2$, thus providing a way to differentiate the $\mathrm{T} 1$ and T2 structures with the same size. From Figs. 4(a) and 4(b), we see that the magnetic coupling is mainly due to the $C$ atoms along the edges of the triangular flake, similar to the situation in a free state. Due to more electrons accumulated at $\mathrm{N}$ atoms, the $\mathrm{N}$ sites are more spin polarized as compared with B sites, and the induced moments are ferromagnetically coupled with $\mathrm{C}$ sites. This can be further confirmed from the partial density of states (PDOS) as shown in Fig. 4(c). We see that the contribution to magnetism follows the order of $\mathrm{C}>$ $\mathrm{N}>$ B. Furthermore, all the induced midgap states are fully spin polarized. Therefore, we can expect that the application of an appropriately tuned bias voltage can therefore produce fully spin-polarized currents, suggesting that the system can be potentially used for spin filtering.

The calculated magnetic moments of two types of triangular graphene-embedded $\mathrm{BN}$ sheets are shown in Fig. 5, where positive $(\alpha-\beta)$ values are for the $\mathrm{T} 1-\mathrm{C}_{\alpha} \mathrm{C}_{\beta}$ system, while negative $(\alpha-\beta)$ values correspond to the T2- $\mathrm{C}_{\alpha} \mathrm{C}_{\beta}$ structure. 


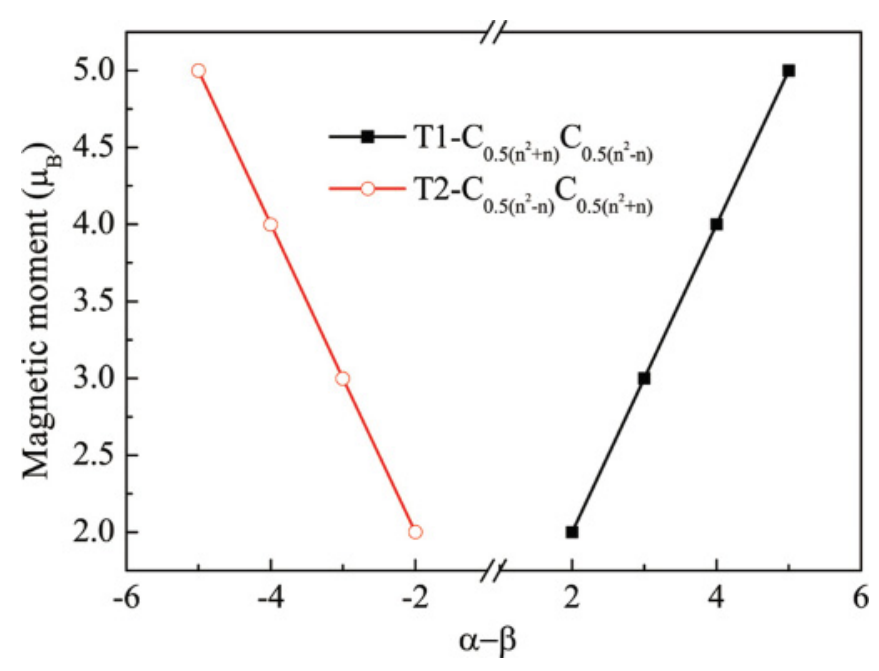

FIG. 5. (Color online) Variation of magnetic moments of T1$\mathrm{C}_{\alpha} \mathrm{C}_{\beta}$ and T2- $\mathrm{C}_{\alpha} \mathrm{C}_{\beta}$ with $(\alpha-\beta)$.

It is interesting to see that in a graphene flake composed $\alpha+\beta$ carbon atoms $|\alpha-\beta|$ electrons or holes can be introduced to a $\mathrm{BN}$ sheet when embedded, depending on the nature of bonding with B or N. Full spin polarizations make the system magnetic with the moment of $|\alpha-\beta| \mu_{B}$ per unit cell. So by tuning the sizes of the triangle graphene flakes, the magnetic moments of T1- and T2-graphene-impregnated BN sheet can be controlled accurately.

\section{CONCLUSIONS}

To modulate the electronic and magnetic properties of an impregnated BN sheet while keeping its 2D planar geometry, we performed first-principles calculations by embedding graphene flakes in the BN sheets. Following conclusions can be drawn: (1) The energy gap reduces with the increase in the flake size, and the system can be tuned to have direct or indirect band gap. (2) When the flake has a hexagonal shape, the hybrid sheet is isoelectronic with the pristine $\mathrm{BN}$ sheet as they have the same number of electrons. However, when the flake has a triangular shape, the situation is very different. If the embedding is through C-N (C-B) bonds, the system becomes hole injected (electron injected). (3) Triangular embedding can induce fully spin-polarized states, which locate close to the conductance bands in hole-injecting case and to the valence bands in electron-injecting case. (4) Magnetism of triangular graphene flakes can be retained when embedded in a BN sheet, and the Lieb theorem remains valid. (5) The $\mathrm{B}$ or $\mathrm{N}$ atoms bonded with the triangular grpahene flakes are ferromagnetically polarized. Our study clearly shows that graphene flakes are promising candidates for modulating the electronic and magnetic properties of $\mathrm{BN}$ sheets.

\section{ACKNOWLEDGMENTS}

This work is partially supported by the National Natural Science Foundation of China (Grant Nos. NSFC-21173007 and NSFC-10874007), from the National Grand Fundamental Research 973 Program of China (2012CB921404), and from the United States Department of Energy.
*Corresponding author:sunqiang@pku.edu.cn

${ }^{1}$ K. S. Novoselov, A. K. Geim, S. V. Morozov, D. Jiang, Y. Zhang, S. V. Dubonos, I. V. Grigorieva, and A. A. Firsov, Science 306, 666 (2004).

${ }^{2}$ C. Berger, Z. Song, X. Li, X. Wu, N. Brown, C. Naud, D. Mayou, T. Li, J. Hass, A. N. Marchenkov, E. H. Conrad, P. N. First, and W. A. de Heer, Science 312, 1191 (2006).

${ }^{3}$ A. K. Geim and K. S. Novoselov, Nat. Mater. 6, 183 (2007).

${ }^{4}$ M. I. Katsnelson, K. S. Novoselov, and A. K. Geim, Nat. Phys. 2, 620 (2006).

${ }^{5}$ B. Huard, J. A. Sulpizio, N. Stander, K. Todd, B. Yang, and

D. Goldhaber-Gordon, Phys. Rev. Lett. 98, 236803 (2007).

${ }^{6}$ K. S. Novoselov, A. K. Geim, S. V. Morozov, D. Jiang, M. I. Katsnelson, I. V. S. Grigorieva, V. Dubonos, and A. A. Firsov, Nature 438, 197 (2005).

${ }^{7}$ Y. B. Zhang, Y. W. Tan, H. L. Stormer, and P. Kim, Nature 438, 201 (2005).

${ }^{8}$ K. S. Novoselov, E. McCann, S. V. Morozov, V. I. Fal'ko, M. I. Katsnelson, U. Zeitler, D. Jiang, F. Schedin, and A. K. Geim, Nat. Phys. 2, 177 (2006).

${ }^{9}$ E. McCann and V. I. Fal'ko, Phys. Rev. Lett. 96, 086805 (2006).

${ }^{10}$ A. K. Geim, Science 324, 1530 (2009).

${ }^{11}$ W. Q. Han, L. Wu, Y. Zhu, K. Watanabe, and T. Taniguchi, Appl. Phys. Lett. 93, 223103 (2008).

${ }^{12}$ D. Pacilé, J. C. Meyer, Ç. O. Girit, and A. Zettl, Appl. Phys. Lett. 92, 133107 (2008).
${ }^{13}$ M. Topsakal, E. Aktürk, and S. Ciraci, Phys. Rev. B 79, 115442 (2009).

${ }^{14}$ J. Zhou, Q. Wang, Q. Sun, and P. Jena, Phys. Rev. B 81, 085442 (2010).

${ }^{15}$ S. Azevedo, J. R. Kaschny, C. M. C. de Castilho, and F. de B. Mota, Eur. Phys. J. B 67, 507 (2009).

${ }^{16}$ Y. G. Zhou, J. Xiao-Dong, Z. G. Wang, H. Y. Xiao, F. Gao, and X. T. Zu, Phys. Chem. Chem. Phys. 12, 7588 (2010).

${ }^{17}$ L. J. Ci, L. Song, C. H. Jin, D. Jariwala, D. X. Wu, Y. J. Li, A. Srivastava, Z. F. Wang, K. Storr, L. Balicas, F. Liu, and P. M. Ajayan, Nat. Mater. 9, 430 (2010).

${ }^{18}$ B. Xu, Y. H. Lu, Y. P. Feng, and J. Y. Lin, J. Appl. Phys. 108, 073711 (2010).

${ }^{19}$ S. Cahangirov and S. Ciraci, Phys. Rev. B 83, 165448 (2011).

${ }^{20}$ E. H. Lieb, Phys. Rev. Lett. 62, 1201 (1989).

${ }^{21}$ X. Wei, M.-S. Wang, Y. Bando, and D. Golberg, ACS Nano 5, 2916 (2011).

${ }^{22}$ J. P. Perdew, K. Burke, and M. Ernzerhof, Phys. Rev. Lett. 77, 3865 (1996).

${ }^{23}$ B. Delley, J. Chem. Phys. 92, 508 (1990); 113, 7756 (2000).

${ }^{24}$ H. J. Monkhorst and J. D. Pack, Phys. Rev. B 13, 5188 (1976).

${ }^{25}$ M. S. Si and D. S. Xue, Phys. Rev. B 75, 193409 (2007).

${ }^{26}$ Y. Liu, S. Bhowmick, and B. I. Yakobson, Nano Lett. 11, 3113 (2011).

${ }^{27}$ N. Berseneva, A. Krasheninnikov, and R. M. Nieminen, Phys. Rev. Lett. 107, 035501 (2011). 